\section{Prevalência e fatores associados ao acesso a medicamentos pela população idosa em uma capital do sul do Brasil: um estudo de base populacional}

\author{
Prevalence and factors associated with access \\ to medication among the elderly in a city in \\ southern Brazil: a population-based study
}

\author{
Marina Meneses Aziz 1 \\ Maria Cristina Calvo 1 \\ Ione Jayce Ceola Schneider 1 \\ André Junqueira Xavier 2 \\ Eleonora d'Orsi 1
}

\footnotetext{
1 Programa de Pósgraduação em Saúde Coletiva, Universidade Federal de Santa Catarina, Florianópolis, Brasil. 2 Universidade do Sul de Santa Catarina Florianópolis, Brasil.

Correspondência M. M. Aziz

Programa de Pós-graduação em Saúde Coletiva,

Universidade Federal de Santa Catarina.

Rua Prof. José Brasilício de Souza 100, apto. 703, Florianópolis, SC 88036-530, Brasil.

marinamaziz@gmail.com
}

\section{Abstract}

This study aimed to describe the prevalence of access to prescription drugs for the elderly and associated factors in Florianópolis, Santa Catarina State, Brazil. A cross-sectional population-based household survey was performed in a sample of 1,469 individuals (non-response rate: 10.8\%). The data were analyzed with Poisson regression, from two perspectives: access to medicines in general and access to medicines through the Unified National Health System (SUS). The results were, respectively, 95.8\% (95\%CI: 94.7-96.8) and 50.3\% (95\%CI: 47.7-52.8). Lower access to medicines was associated with functional dependence, chronic illness, and at least one medical visit in the previous three months. Access to medicines through the National Health System was associated with black or mixed race, younger age, lower schooling, lower income, more chronic diseases, lack of private health insurance, and at least one medical visit in the previous three months. The results show that the National Health System has been successful in promoting universal access to medicines in Florianópolis.

Aged; Health Services Accessibility; Drug Utilization; Unified Health System; Cross-Sectional Studies

\section{Introdução}

O padrão demográfico brasileiro vem se modificando desde a década de 1940, com crescimento mais lento do número de crianças e adolescentes, paralelamente a um aumento da população em idade ativa e de pessoas idosas. Estima-se que o grupo etário de 60 anos ou mais duplicará, em termos absolutos, no período de 2000 a 2020, ao passar de 13,9 para 28,3 milhões, elevando-se, em 2050, para 64 milhões ${ }^{1}$.

O envelhecimento populacional no Brasil tem gerado novas demandas, que requerem um planejamento dos sistemas de saúde públicos para atender às necessidades específicas dos idosos. Grande parte dos idosos possui alguma doença crônica, o que leva a uma maior utilização de serviços de saúde e a um elevado consumo de medicamentos. Estima-se que a maioria dos idosos utilize, pelo menos, um medicamento, e cerca de um terço deles consuma cinco ou mais simultaneamente. A média de produtos usados por pessoa oscila entre dois e cinco $^{2}$.

Uma prioridade nas políticas de saúde atuais, como a Política Nacional de Medicamentos ${ }^{3}$ e o Pacto pela Saúde 4, é garantir, principalmente para essa população, o acesso a medicamentos essenciais com qualidade e segurança. $\mathrm{O}$ acesso se concretiza com a utilização do medicamento, portanto, conceitua-se acesso como um fator mediador entre a capacidade de um sistema de 
saúde de oferecer o produto e o consumo real deste produto pela população ${ }^{5}$.

No Brasil, existem formas de organização/ financiamento mistas para o acesso a medicamentos: fornecimento de medicamentos pela rede pública de saúde, através do Sistema Único de Saúde (SUS), e pelo sistema privado, constituído de seguros de saúde ou por pagamento direto 6. Segundo a Pesquisa Nacional por Amostra de Domicílios (PNAD) 1998, o gasto médio com medicamentos referidos pelos idosos foi igual a $23 \%$ do valor do salário mínimo 7 . A prestação de serviços pela rede pública torna-se a única alternativa para a assistência à saúde e o acesso aos medicamentos para uma grande parcela da população que não possui condições financeiras de arcar com seus custos 8 .

$\mathrm{O}$ acesso aos medicamentos é um fator determinante no cumprimento do tratamento prescrito. O não acesso a medicamentos pode levar a descompensação clínica e aumento dos gastos com a atenção secundária e terciária ${ }^{9}$. As barreiras ao acesso a esses produtos, especialmente de ordem financeira, figuram entre os principais motivos apontados pelos usuários para a subutilização dos medicamentos prescritos. Estima-se que um quarto dos idosos subutilize medicamentos em virtude dos altos custos deles 10 .

Apesar das diferentes metodologias, as prevalências de acesso aos medicamentos em estudos populacionais variam de $87 \%$ a $90 \% 8,9,11,12$. A medida de acesso a medicamentos é importante, pois indica a qualidade e a resolutividade de um sistema de saúde, além de contribuir para a construção de estratégias de intervenção e reorientação das políticas de medicamentos.

Estudos populacionais sobre acesso a medicamentos são muito raros no Brasil, e a maioria se ateve a medir o acesso de idosos residentes nas áreas de abrangência das Unidades Básicas de Saúde 9,12. Na realização de estudos populacionais de base domiciliar obtêm-se informações reais sobre como a população está tendo acesso e usando os medicamentos, representando e caracterizando o sistema de saúde como um todo ${ }^{8}$.

Portanto, o presente estudo tem o objetivo de descrever a prevalência e fatores associados ao acesso da população idosa, residente na área urbana do Município de Florianópolis, Santa Catarina, aos medicamentos que lhe foram prescritos. Além disso, verifica a associação entre o acesso a medicamentos através do SUS com as características sociodemográficas, grau de dependência, presença de doenças crônicas e utilização de serviços de saúde.

\section{Métodos}

Foi realizado um estudo transversal populacional de base domiciliar com idosos do Município de Florianópolis. O estudo faz parte de um inquérito abrangente sobre as condições de saúde das pessoas idosas do Município de Florianópolis, realizado em 2009/2010 denominado EpiFloripa Idoso. A pesquisa foi financiada pelo Conselho Nacional de Desenvolvimento Cientifico e Tecnológico (CNPq) e aprovado pelo Comitê de Ética em Pesquisa da Universidade Federal de Santa Catarina (UFSC).

O Município de Florianópolis é a capital do Estado de Santa Catarina, com população estimada para o ano de 2009 13, de 408.163 habitantes, sendo 44.460 pertencentes à faixa etária com idade igual ou superior a 60 anos (18.844 do sexo masculino e 25.616 do sexo feminino), representando $10,8 \%$ da população total. A esperança de vida ao nascer era de 72,8 anos e o município apresentava um Índice de Desenvolvimento $\mathrm{Hu}$ mano Municipal (IDH-M) de 0,875 em 2000, colocando o município na 4 a posição dentre todos os municípios brasileiros 14 .

O tamanho da amostra foi calculado para estimar a prevalência a partir de uma amostra casual simples, multiplicada por 2 (conglomerado em 2 estágios), e acréscimos de $20 \%$ para perdas previstas e $15 \%$ para controle de fatores de confusão. Para tal, foi usado o programa Epi Info, versão 6.04 (Centers for Disease Control and Prevention, Atlanta, Estados Unidos), com os seguintes parâmetros: tamanho da população igual a 44.460, prevalência para o desfecho desconhecida (50\%), nível de $95 \%$ de confiança, erro amostral igual a 4 pontos percentuais, resultando em uma amostra mínima de 1.599 pessoas.

O processo de seleção da amostra foi realizado por conglomerados em dois estágios. No primeiro estágio, todos os 420 setores censitários urbanos da cidade foram colocados em ordem crescente de renda média mensal do chefe da família, sorteando-se sistematicamente 80 destes setores (oito setores em cada decil de renda).

As unidades de segundo estágio foram os domicílios. Uma etapa de atualização do número de domicílios em cada setor (arrolamento) fez-se necessária uma vez que o Censo mais recente havia sido realizado em 2000. Para tanto, os supervisores do estudo percorreram cada um dos setores censitários sorteados e procederam à contagem de todos os domicílios habitados, obedecendo às normas do Instituto Brasileiro de Geografia e Estatística (IBGE). O número de domicílios nos setores variou de 61 a 725 .

Setores com menos de 150 domicílios foram agrupados e setores com mais de 500 domicílios 
foram divididos, respeitando o decil de renda correspondente, com a finalidade de diminuir o coeficiente de variação do número de domicílios por setor. Tal procedimento resultou em 83 setores censitários e reduziu o coeficiente de variação inicial de $52,7 \%$ para $35,2 \%$.

Segundo o IBGE 15, o número médio de moradores por domicílio equivale a 3,1. Como a faixa etária de interesse da pesquisa corresponde a aproximadamente $11 \%$ da população, obtêm-se em média, por setor censitário, 102 pessoas na faixa etária de interesse ou 1 idoso a cada três domicílios. Estimou-se, portanto, que deveriam ser visitados cerca de 60 domicílios por setor censitário, para se encontrarem os 20 idosos. Esses domicílios foram sorteados de forma sistemática e foram considerados elegíveis para serem entrevistados todos os idosos residentes nos domicílios sorteados.

Foram considerados como perda os idosos não localizados após quatro visitas, sendo pelo menos uma no período noturno e uma no final de semana, além daqueles que se encontravam impossibilitados de responder por motivo de viagem. E recusas, aqueles que se negaram a responder o questionário por opção pessoal.

A coleta de dados foi realizada por meio de um questionário padronizado e pré-testado aplicado na forma de entrevistas face a face, utilizando-se o Personal Digital Assistants (PDA).

O trabalho de campo foi realizado entre setembro de 2009 e junho de 2010. As entrevistas foram realizadas por entrevistadoras do sexo feminino com nível médio completo de escolaridade ou mais, devidamente treinadas sobre os procedimentos da pesquisa. O estudo-piloto incluiu 99 idosos residentes em setores não amostrados para a pesquisa. O trabalho de campo foi supervisionado por estudantes de cursos de pósgraduação.

Houve verificação semanal da consistência dos dados e controle de qualidade por meio de aplicação por telefone de um questionário reduzido, em $10 \%$ das entrevistas selecionadas aleatoriamente.

Os dados sobre medicamentos foram coletados sob duas perspectivas: a do acesso a medicamentos (indivíduos que usaram medicamentos nos 30 dias anteriores à entrevista, e, portanto, tiveram acesso a estes de alguma forma) e do não acesso a medicamentos (indivíduos que relataram terem deixado de tomar algum medicamento de que precisavam, sendo incluídos somente os casos em que a não utilização ocorreu por não acesso).

No caso de o entrevistado ter tido acesso ao medicamento, foi perguntado também quem indicou o medicamento (repetiu uma receita an- tiga; a pessoa mesmo decidiu tomar o remédio; médico ou dentista do SUS; médico ou dentista particular ou do plano de saúde; farmacêutico ou o balconista da farmácia; enfermeiro, fisioterapeuta ou outro profissional da saúde; parentes, vizinhos ou amigos) e como o entrevistado o obteve (farmácia do posto/Policlínica/Hospital SUS; comprou; comprou e ganhou uma parte).

Nos casos em que o entrevistado não teve acesso a algum medicamento, foi perguntado de quem foi a indicação para a utilização do medicamento (mesmas opções de resposta acima), se era de uso contínuo ou esporádico, o motivo da não obtenção do medicamento (não tinha na farmácia do SUS; não tinha dinheiro; falta de receita médica) e atitudes frente a essa situação (nenhuma atitude; procurou novamente o médico; procurou outro posto de saúde; procurou um advogado).

Foram definidas duas variáveis de desfecho: "acesso a medicamentos" e "acesso a medicamentos pelo SUS”.

A variável desfecho "acesso a medicamentos" foi definida de forma dicotômica: indivíduos que obtiveram acesso a todos os medicamentos prescritos ou indivíduos que não obtiveram acesso aos medicamentos prescritos, incluindo os com acesso parcial. Considerando que foram utilizadas as seguintes perguntas para a composição dessa variável: 1) "Nos últimos 30 dias, o sr.(a) usou algum remédio?" e 2) "Neste período de 30 dias o(a) sr. (a) deixou de tomar algum remédio de que precisava?", as opções foram: acesso total indivíduos que obtiveram acesso a todos os medicamentos prescritos (resposta "Sim" na questão 1 e "Não" na questão 2); acesso parcial - indivíduos que obtiveram acesso a parte dos medicamentos prescritos (resposta "Sim" na questão 1 e "Sim" na questão 2); não acesso - indivíduos que não obtiveram acesso a nenhum dos medicamentos prescritos (resposta "Não" na questão 1 e "Sim" na questão 2).

A segunda variável de desfecho "acesso a medicamentos pelo SUS” também foi definida de forma dicotômica: indivíduos que relataram conseguir pelo menos um de seus medicamentos pelo SUS versus aqueles que os obtiveram por qualquer outro meio (compra ou amostra grátis). Essa variável foi obtida da mesma forma que a variável anterior, sendo que, para verificar a principal forma de obtenção do medicamento, foi utilizada a pergunta do questionário "Como conseguiu este remédio?”. Considerando que o idoso pode ter utilizado de 1 até 28 medicamentos prescritos por médico nos 30 dias anteriores à entrevista (conforme dados da pesquisa), e cada um destes medicamentos foi obtido de uma forma específica (SUS ou compra), o indivíduo foi 
considerado na categoria "acesso a medicamentos pelo SUS" se conseguiu obter, dentre todos os medicamentos utilizados, pelo menos 1 destes pelo SUS.

As variáveis independentes analisadas foram as características socioeconômicas - sexo, cor da pele autorreferida, excluindo-se amarelos e indígenas (branca; parda; preta), idade, grau de escolaridade em anos de estudo e renda familiar per capita. O grau de dependência foi verificado pela presença ou não de cuidador, além da capacidade funcional do idoso, avaliada através de uma escala de 15 atividades da vida diária, incluindo atividades pessoais (p. ex.: banhar-se, vestir-se, ir ao banheiro em tempo) e instrumentais (p. ex.: preparar refeições, subir escadas, pegar ônibus) e categorizada como: 1) ausência de dependência - incapacidade/dificuldade em nenhuma das atividades; 2) presença de dependência leve - incapacidade/dificuldade em 1 a 3 atividades; 3) presença de dependência moderada/grave - incapacidade/dificuldade em 4 ou mais atividades 16. Também foram analisadas as variáveis dicotômicas: presença de doenças crônicas, possuir ou não plano de saúde; realização de consulta com médico nos últimos 3 meses, além do número de medicamentos utilizados (um; dois a três; quatro a cinco; seis ou mais).

Todas as variáveis do estudo foram analisadas de forma descritiva por meio de frequência absoluta e relativa. A estatística descritiva incluiu cálculos de proporções e intervalos de 95\% de confiança para variáveis categóricas, e médias e desvios-padrão para as numéricas.

Para testar a associação entre os desfechos e as variáveis independentes, foi realizada análise bruta e multivariada por meio de regressão de Poisson, estimando-se razões de prevalência brutas e ajustadas, com intervalos de 95\% de confiança e valor de $\mathrm{p}$ (obtido por meio do teste de Wald).

As variáveis que apresentaram valor de $\mathrm{p}<$ 0,20 na análise bruta foram selecionadas para entrarem no modelo múltiplo, permanecendo nos modelos se atingissem $\mathrm{p}<0,05 \mathrm{e} / \mathrm{ou}$ ajustassem o modelo.

Utilizou-se o pacote estatístico Stata 9.0 (Stata Corp., College Station, Estados Unidos) e todas as análises consideraram o efeito do desenho amostral por meio do comando svy, projetado para a análise de dados provenientes de amostras complexas.

\section{Resultados}

Nos domicílios sorteados foram encontrados 1.911 idosos elegíveis, sendo efetivamente entrevistados 1.705 idosos, resultando em uma taxa de resposta de $89,2 \%$. Os principais motivos de perdas ( $\mathrm{n}=206$ ): "não tinha ninguém em casa", "o idoso estava viajando", "marcou com a entrevistadora e não compareceu”, "estava de férias”, "estava muito doente", "tinha cachorro bravo no terreno". Houve 3 perdas por motivo de hospitalização do idoso no momento da entrevista, não afetando os resultados. Os principais motivos de recusas foram: "não quis dar entrevista", "entrevista muito longa", "sem tempo para responder a entrevista", "acha perda de tempo responder entrevistas", "não acredita em pesquisas".

$\mathrm{O}$ controle de qualidade verificou que a reprodutibilidade de algumas questões utilizadas no estudo foi considerada satisfatória, com valores de kappa variando entre 0,6 a 0,9, para variáveis selecionadas, sendo que a maioria apresentou concordância boa a excelente.

Somente foram analisadas as respostas de indivíduos que utilizaram pelo menos um dos medicamentos prescritos por médico/dentista, totalizando 1.469 pessoas, ou seja, 85,9\% da amostra total (Tabela 1). Desse total, 3 indivíduos não responderam onde obtiveram seus medicamentos, resultando em 1.466 pessoas (Tabela 2), causando uma diferença entre os $\mathrm{n}$ das duas tabelas. As Tabelas 1 e 2 descrevem a amostra, sendo que a maioria desses indivíduos era do sexo feminino $(66,6 \%)$ e considerava a cor da sua pele como branca $(87,8 \%)$. Em relação à idade, boa parte tinha entre 60 e 69 anos (46,8\%) e estudou na escola por um período de 4 anos ou menos $(44,3 \%)$. A renda familiar per capita média esteve em torno de $\mathrm{R} \$ 1.392,00$.

Possuíam algum cuidador 9,8\% dos idosos, sendo que a grande parte deles $(41,3 \%)$ possuía dependência leve, ou seja, incapacidade/dificuldade de realizar de 1 a 3 atividades de uma escala de 15 atividades da vida diária. Quanto à utilização dos serviços de saúde, $65,5 \%$ possuíam plano de saúde e $76,7 \%$ dos entrevistados relataram ter consultado com o médico nos 3 meses anteriores à entrevista.

Somente $4,3 \%$ dos entrevistados não possuíam nenhuma doença crônica, sendo que a maioria deles relataram possuir de 2 a 4 doenças crônicas $(57,9 \%)$. Um terço dos entrevistados relatou que utilizou de 2 a 3 medicamentos nos 30 dias anteriores à entrevista (32,9\%) e a média foi de 4,40 medicamentos por pessoa (IC95\%: 4,26$4,56)$. Porém, chamou atenção a grande proporção de idosos que utilizaram 6 ou mais medicamentos $(27,3 \%)$. 
Tabela 1

Características da amostra de idosos e prevalência de acesso a medicamentos prescritos de acordo com variáveis demográficas, socioeconômicas, grau de dependência, morbidades, uso de serviços de saúde e medicamentos.

Florianópolis, Santa Catarina, Brasil, 2009/2010.

\begin{tabular}{|c|c|c|c|c|}
\hline \multirow[t]{2}{*}{ Variável } & \multicolumn{2}{|c|}{ Amostra } & \multirow[t]{2}{*}{ Prevalência (IC95\%) } & \multirow[t]{2}{*}{ Valor de $p$ ** } \\
\hline & $n$ * & $\%$ & & \\
\hline $\operatorname{Sexo}(n=1.469)$ & & & & 0,064 \\
\hline Masculino & 491 & 33,4 & $97,1(95,4-98,9)$ & \\
\hline Feminino & 978 & 66,6 & $95,1(93,6-96,5)$ & \\
\hline Cor da pele autorreferida $(n=1.431)$ & & & & 0,432 \\
\hline Brancos & 1.256 & 87,8 & $96,0(94,8-97,2)$ & \\
\hline Pardos & 106 & 7,4 & $93,4(87,6-99,2)$ & \\
\hline Pretos & 69 & 4,8 & $95,6(90,5-100,8)$ & \\
\hline Faixa etária em anos $(n=1.469)$ & & & & $0,439 * \star \star$ \\
\hline $60-69$ & 688 & 46,8 & $94,9(93,1-96,7)$ & \\
\hline $70-79$ & 560 & 38,1 & $97,1(95,6-98,6)$ & \\
\hline 80 ou mais & 221 & 15,1 & $95,0(92,5-97,5)$ & \\
\hline Escolaridade em anos de estudo $(n=1.459)$ & & & & $0,298 * \star \star$ \\
\hline$\geq 12$ & 337 & 23,1 & $96,4(94,4-98,5)$ & \\
\hline $9-11$ & 203 & 13,9 & $96,1(93,3-98,8)$ & \\
\hline $5-8$ & 272 & 18,6 & $96,3(93,9-98,7)$ & \\
\hline$\leq 4$ & 647 & 44,3 & $95,0(93,2-96,9)$ & \\
\hline Renda familiar per capita $(n=1.469)$ & & & & $0,136 * \star \star$ \\
\hline Quartil 4 & 365 & 24,8 & $97,5(95,8-99,3)$ & \\
\hline Quartil 3 & 369 & 25,1 & $96,2(94,4-98,0)$ & \\
\hline Quartil 2 & 367 & 25,0 & $93,2(90,0-96,4)$ & \\
\hline Quartil 1 & 368 & 25,1 & $96,2(94,3-98,1)$ & \\
\hline Possui cuidador $(n=1.468$ ) & & & & 0,403 \\
\hline Não & 1.324 & 90,2 & $95,9(94,7-97,1)$ & \\
\hline Sim & 144 & 9,8 & $94,4(90,7-98,2)$ & \\
\hline Capacidade funcional $(n=1.469)$ & & & & $<0,001$ \\
\hline Ausência de dependência & 357 & 24,3 & $98,0(96,4-99,6)$ & \\
\hline Dependência leve & 606 & 41,3 & $97,0(95,7-98,4)$ & \\
\hline Dependência moderada/grave & 506 & 34,4 & $92,7(90,2-95,2)$ & \\
\hline Doença crônica $(n=1.450)$ & & & & $<0,001 \star \star \star *$ \\
\hline $0-1$ & 282 & 19,5 & $97,9(96,2-99,5)$ & \\
\hline $2-4$ & 840 & 57,9 & $97,0(95,8-98,2)$ & \\
\hline 5 ou mais & 328 & 22,6 & $91,2(87,7-94,6)$ & \\
\hline Plano de Saúde privado $(n=1.469)$ & & & & 0,870 \\
\hline $\operatorname{Sim}$ & 962 & 65,5 & $95,8(94,5-97,2)$ & \\
\hline Não & 507 & 34,5 & $95,7(93,5-97,8)$ & \\
\hline Consulta médica nos últimos 3 meses $(n=1.469)$ & & & & 0,022 \\
\hline $\operatorname{Sim}$ & 1.127 & 76,7 & $95,1(93,7-96,6)$ & \\
\hline Não & 342 & 23,3 & $97,9(96,5-99,4)$ & \\
\hline Número de medicamentos utilizados $(n=1.469)$ & & & & $0,124 * \star *$ \\
\hline Um & 183 & 12,5 & $95,1(91,4-98,7)$ & \\
\hline $2-3$ & 483 & 32,9 & $97,5(96,1-98,9)$ & \\
\hline $4-5$ & 402 & 27,4 & $95,5(93,4-97,7)$ & \\
\hline 6 ou mais & 401 & 27,3 & $94,3(91,6-96,9)$ & \\
\hline
\end{tabular}

IC95\%: intervalo de 95\% de confiança.

* Número de entrevistados com pelo menos 1 medicamento prescrito por médico;

** Valor de $\mathrm{p}$ no teste $\chi^{2}$;

$\star \star \star$ Valor de $p$ de tendência linear. 
Características da amostra de idosos e prevalência de acesso a medicamentos pelo SUS de acordo com variáveis demográficas, socioeconômicas, grau de dependência, morbidades, uso de serviços de saúde e medicamentos. Florianópolis, Santa Catarina, Brasil, 2009/2010.

\begin{tabular}{|c|c|c|c|c|}
\hline \multirow[t]{2}{*}{ Variável } & \multicolumn{2}{|c|}{ Amostra } & \multirow[t]{2}{*}{ Prevalência (IC95\%) } & \multirow[t]{2}{*}{ Valor de $p$ ** } \\
\hline & $n$ * & $\%$ & & \\
\hline $\operatorname{Sexo}(n=1.466)$ & & & & 0,555 \\
\hline Masculino & 490 & 33,4 & $49,2(43,5-54,9)$ & \\
\hline Feminino & 976 & 66,6 & $50,8(45,1-56,5)$ & \\
\hline Cor da pele autorreferida $(n=1.428)$ & & & & $<0,001$ \\
\hline Brancos & 1.253 & 87,7 & $47,2(42,2-52,3)$ & \\
\hline Pardos & 106 & 7,4 & $71,7(61,9-81,5)$ & \\
\hline Pretos & 69 & 4,8 & $73,9(64,2-83,6)$ & \\
\hline Faixa etária em anos $(n=1.466)$ & & & & $0,092 * \star \star$ \\
\hline $60-69$ & 686 & 46,8 & $51,2(45,7-56,6)$ & \\
\hline $70-79$ & 559 & 38,1 & $52,2(46,2-58,3)$ & \\
\hline 80 ou mais & 221 & 15,1 & $42,5(33,1-51,9)$ & \\
\hline Escolaridade em anos de estudo $(n=1.456)$ & & & & 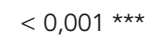 \\
\hline$\geq 12$ & 336 & 23,1 & $19,9(13,9-25,9)$ & \\
\hline $9-11$ & 202 & 13,9 & $37,1(30,7-43,5)$ & \\
\hline $5-8$ & 272 & 18,7 & $54,4(46,3-62,5)$ & \\
\hline$\leq 4$ & 646 & 44,4 & $68,3(63,8-72,7)$ & \\
\hline Renda familiar per capita $(n=1.466)$ & & & & $<0,001 * \star \star$ \\
\hline Quartil 4 & 365 & 24,9 & $18,1(13,7-22,5)$ & \\
\hline Quartil 3 & 368 & 25,1 & $46,7(40,2-53,2)$ & \\
\hline Quartil 2 & 366 & 25,0 & $68,3(63,1-73,5)$ & \\
\hline Quartil 1 & 367 & 25,0 & $67,8(61,5-74,2)$ & \\
\hline Possui cuidador $(n=1.465)$ & & & & 0,001 \\
\hline Não & 1.321 & 90,2 & $48,8(43,9-53,8)$ & \\
\hline Sim & 144 & 9,8 & $63,9(54,1-73,7)$ & \\
\hline Capacidade funcional $(n=1.466)$ & & & & $<0,001$ \\
\hline Ausência de dependência & 355 & 24,2 & $43,4(36,4-50,3)$ & \\
\hline Dependência leve & 606 & 41,3 & $43,9(37,9-49,8)$ & \\
\hline Dependência moderada/grave & 505 & 34,5 & $62,8(56,8-68,8)$ & \\
\hline Doença crônica $(n=1.447)$ & & & & $<0,001^{\star \star \star}$ \\
\hline $0-1$ & 280 & 19,3 & $35,7(28,6-42,8)$ & \\
\hline $2-4$ & 840 & 58,1 & $49,8(44,3-55,2)$ & \\
\hline 5 ou mais & 327 & 22,6 & $63,0(55,7-70,3)$ & \\
\hline Plano de saúde privado $(n=1.466)$ & & & & $<0,001$ \\
\hline $\operatorname{Sim}$ & 960 & 65,5 & $34,5(29,6-39,4)$ & \\
\hline Não & 506 & 34,5 & $80,2(76,0-84,5)$ & \\
\hline Consulta médica nos últimos 3 meses $(n=1.466)$ & & & & $<0,001$ \\
\hline $\operatorname{Sim}$ & 1.125 & 76,7 & $54,2(48,9-59,5)$ & \\
\hline Não & 341 & 23,3 & $37,2(30,4-44,1)$ & \\
\hline Número de medicamentos utilizados $(n=1.466)$ & & & & $<0,001 * \star \star$ \\
\hline 1 & 180 & 12,3 & $38,3(28,7-48,0)$ & \\
\hline $2-3$ & 483 & 33,0 & $40,2(34,1-46,2)$ & \\
\hline $4-5$ & 402 & 27,4 & $52,5(44,9-60,1)$ & \\
\hline 6 ou mais & 401 & 27,3 & $65,6(58,6-72,5)$ & \\
\hline
\end{tabular}

IC95\%: intervalo de $95 \%$ de confiança.

* Número de entrevistados com pelo menos 1 medicamento prescrito por médico, que responderam onde obtiveram seus medicamentos;

** Valor de $p$ no teste $\chi^{2}$;

$\star \star \star$ Valor de $p$ de tendência linear. 
Prevalência e fatores associados ao acesso a medicamentos

A prevalência de acesso total aos medicamentos prescritos pelo médico/dentista foi de $95,8 \%$ (IC95\%: 94,7-96,8), restando 4,2\% (IC95\%: 3,2$5,2)$ de indivíduos que não tiveram acesso a algum medicamento.

Dos medicamentos que não foram obtidos pelos idosos entrevistados, 74,2\% (IC95\%: 63,085,4 ) eram de uso contínuo e 93,5\% foram prescritos pelo médico. A maioria relatou que não utilizou o medicamento, pois não tinha dinheiro para comprá-lo (53,8\%; IC95\%: 37,5-70,2), porém alguns relataram a falta do medicamento na farmácia do SUS (25,6\%; IC95\%: 11,3-40,0) e a falta de receita médica (20,5\%; IC95\%: 7,2-33,8).

Quando questionados sobre a atitude tomada quando não conseguiram o medicamento, 70,8\% (IC95\%: 57,5-84,2) dos idosos não fizeram nada, 22,9\% (IC95\%: 10,6-35,2) procuraram o médico novamente e 6,2\% (IC95\%: 0,8-13,3) procuraram outro posto de saúde.

A prevalência de acesso aos medicamentos prescritos aos idosos (Tabela 1) não apresentou diferença significativa entre os sexos, cor da pele, faixa etária, escolaridade, renda familiar per capita, número de medicamentos utilizados, possuir cuidador ou plano de saúde privado. $\mathrm{O}$ acesso aos medicamentos foi menor para os idosos com maior grau de dependência e maior número de doenças crônicas $(\mathrm{p}<0,001)$. Aqueles que se consultaram com o médico nos 3 meses anteriores à entrevista $(\mathrm{p}=0,022)$ tiveram uma menor probabilidade de ter acesso a medicamentos comparando com quem não realizou consulta médica.

Somente as variáveis "sexo", "renda”, "capacidade funcional”, "número de doenças crônicas”, "ter realizado consulta médica nos últimos 3 meses" e "número de medicamentos utilizados" tiveram valores de $\mathrm{p}<0,2$ na análise bivariada, e, portanto, foram adicionadas na análise bruta e ajustada para verificar a associação da variável "acesso a medicamentos" com essas variáveis independentes (Tabela 3). Na análise bruta, somente as variáveis "capacidade funcional", "número de doenças crônicas" e "ter realizado consulta médica nos últimos 3 meses" obtiveram valores de $\mathrm{p}<0,05$, e o mesmo permaneceu na análise ajustada.

Portanto, pode-se dizer que existe associação entre o fato do idoso ter menos acesso aos medicamentos prescritos quando possui um grau de dependência maior, quando tem um maior número de doenças crônicas e quando se consultou com médico.
Prevalência e fatores associados ao acesso a medicamentos pelo SUS

Dos 1.469 entrevistados que utilizaram pelo menos um dos medicamentos prescritos pelo médico/dentista, 1.466 responderam qual foi a forma de obtenção desses medicamentos, totalizando $85,7 \%$ da amostra total.

Considerando-se pelo menos um dos medicamentos utilizados pelo idoso sendo obtido pelo SUS, 50,3\% (IC95\%: 47,7-52,8) dos idosos relataram obter medicamentos por essa via, sendo que o restante da amostra compra os medicamentos que lhe são prescritos.

A Tabela 2 mostra a prevalência de acesso a medicamentos pelo SUS em relação às variáveis independentes. Indivíduos que consideraram a cor da pele como parda e preta ( $p<0,001)$, com menor escolaridade $(\mathrm{p}<0,001)$ e de menor renda per capita familiar $(\mathrm{p}<0,001)$ utilizaram mais o SUS para ter acesso a medicamentos, assim como indivíduos com a capacidade funcional pior $(\mathrm{p}<0,001)$ e que relataram possuir cuidador $(\mathrm{p}=$ 0,001 ). Também aumenta a prevalência de acesso a medicamentos pelo SUS conforme aumenta o número de doenças crônicas ( $\mathrm{p}<0,001)$, o número de medicamentos utilizados $(\mathrm{p}<0,001)$, bem como em indivíduos que não possuem plano de saúde ( $\mathrm{p}<0,001)$ e que consultaram com médico nos 3 meses anteriores à entrevista $(\mathrm{p}<0,001)$.

A prevalência de acesso aos medicamentos pelo SUS não apresentou diferença significativa entre os sexos ( $p=0,555)$.

A variável "sexo" obteve valor de p > 0,2 na análise bivariada, portanto foi excluída da análise de associação entre a variável "obtenção de medicamento pelo SUS” com as variáveis independentes (Tabela 4). As variáveis "capacidade funcional" e "possui cuidador" tiveram o valor de "p" não significativo ( $\mathrm{p}>0,05)$ na análise ajustada e a variável "número de medicamentos utilizados" foi retirada da análise por ser uma variável colinear com a variável "doença crônica”.

Existe associação entre "obter medicamentos pelo SUS" e referir a "cor da pele" como parda ou preta ( $\mathrm{p}=0,001$ ), mesmo após o ajuste com as variáveis socioeconômicas (renda per capita familiar e anos de estudo). Em relação à faixa etária ( $p=0,002$ ), também foi demonstrada associação entre utilizar menos o SUS para obter medicamentos com o aumento da idade.

A análise ajustada também confirmou a associação entre a maior utilização do SUS e o maior o número de doenças crônicas autorreferidas ( $\mathrm{p}<0,001)$, quando o idoso não possui plano de saúde $(\mathrm{p}<0,001)$ e quando realiza consulta médica $(\mathrm{p}<0,001)$. 
Análise bruta e ajustada da variável dependente "acesso a medicamentos" com variáveis demográficas, socioeconômicas, grau de dependência, morbidades, uso de serviços de saúde e medicamentos. Florianópolis, Santa Catarina, Brasil, $2009 / 2010$

\begin{tabular}{|c|c|c|c|c|}
\hline Variável & $\mathrm{RPb}(\mathrm{IC} 95 \%)$ & Valor de p & $\mathrm{RPa}(\mathrm{IC} 95 \%)$ & Valor de $\mathrm{p}$ \\
\hline Sexo $(n=1.469)$ & & 0,053 & & NS \\
\hline Masculino & 1,00 & & & \\
\hline Feminino & $0,98(0,96-1,00)$ & & & \\
\hline Renda familiar per capita $(n=1.469)$ & & 0,126 & & NS \\
\hline Quartil 4 & 1,00 & & & \\
\hline Quartil 3 & $0,99(0,96-1,01)$ & & & \\
\hline Quartil 2 & $0,95(0,92-0,99)$ & & & \\
\hline Quartil 1 & $0,99(0,96-1,01)$ & & & \\
\hline Capacidade funcional $(n=1.469)$ & & 0,001 & & 0,006 \\
\hline Ausência de dependência & 1,00 & & 1,00 & \\
\hline Dependência leve & $0,99(0,97-1,01)$ & & $1,00(0,97-1,02)$ & \\
\hline Dependência moderada/grave & $0,94(0,92-0,97)$ & & $0,96(0,93-0,99)$ & \\
\hline Doença crônica ( $n=1.450)$ & & $<0,001$ & & 0,002 \\
\hline $0-1$ & 1,00 & & 1,00 & \\
\hline $2-4$ & $0,99(0,97-1,01)$ & & $0,99(0,97-1,01)$ & \\
\hline 5 ou mais & $0,93(0,90-0,97)$ & & $0,94(0,90-0,98)$ & \\
\hline Consulta médica nos últimos 3 meses $(n=1.469)$ & & 0,004 & & 0,015 \\
\hline $\operatorname{Sim}$ & 1,00 & & 1,00 & \\
\hline Não & $1,03(1,01-1,05)$ & & $1,02(1,00-1,04)$ & \\
\hline Número de medicamentos utilizados $(n=1.469)$ & & 0,198 & & NS \\
\hline 1 & 1,00 & & & \\
\hline $2-3$ & $1,02(0,98-1,07)$ & & & \\
\hline $4-5$ & $1,00(0,96-1,05)$ & & & \\
\hline 6 ou mais & $0,99(0,95-1,04)$ & & & \\
\hline
\end{tabular}

IC95\%: intervalo de 95\% de confiança; NS: valor de p não significativo ou > 0,05; RPa: razão de prevalência na análise ajustada; RPb: razão de prevalência na análise bruta.

Excluído: obteve valor de $\mathrm{p}$ na análise bruta $>0,2$.

\section{Discussão}

A prevalência de acesso a medicamentos foi praticamente universal entre os idosos residentes em Florianópolis, não sendo observadas diferenças significativas no acesso segundo sexo, idade, renda ou escolaridade. Foram fatores significativamente associados ao menor acesso, a dependência funcional, o maior número de doenças crônicas e ter realizado consulta com o médico nos três meses anteriores à entrevista. A aquisição de medicamentos pelo SUS foi elevada na população estudada, e significativamente associada a cor da pele parda ou negra, menor idade, pior escolaridade, pior renda, maior número de doenças crônicas, ausência de plano de saúde e realização de consulta médica nos últimos três meses.
O tamanho da amostra e a metodologia da pesquisa garantem que os resultados sejam representativos de toda a população de idosos residentes na zona urbana do Município de Florianópolis.

Para minimizar um possível viés de memória, foram analisadas somente as informações de indivíduos que tiveram pelo menos um dos medicamentos prescrito por médico/dentista, fato que minimiza o esquecimento por serem medicamentos incorporados na rotina diária do idoso. Porém, no caso dos medicamentos aos quais os indivíduos relataram não terem acesso, geralmente não havia a embalagem/bula, fato que pode ter ocasionado um maior viés de memória. 
Tabela 4

Análise bruta e ajustada da variável dependente "acesso a medicamentos pelo SUS" com variáveis demográficas, socioeconômicas, grau de dependência, morbidades, uso de serviços de saúde e medicamentos. Florianópolis, Santa Catarina, Brasil, 2009/2010

\begin{tabular}{|c|c|c|c|c|}
\hline Variável & $\mathrm{RPb}$ (IC95\%) & Valor de $p$ & $\mathrm{RPa}(\mathrm{IC95 \% )}$ & Valor de $\mathrm{p}$ \\
\hline Cor da pele autorreferida $(n=1.428)$ & & $<0,001$ & & 0,001 \\
\hline Brancos & 1,00 & & 1,00 & \\
\hline Pardos & $1,52(1,30-1,77)$ & & $1,17(1,00-1,36)$ & \\
\hline Pretos & $1,56(1,34-1,82)$ & & $1,23(1,07-1,41)$ & \\
\hline Faixa etária em anos $(n=1.466)$ & & 0,159 & & 0,002 \\
\hline $60-69$ & 1,00 & & 1,00 & \\
\hline $70-79$ & $1,02(0,91-1,15)$ & & $0,90(0,82-1,00)$ & \\
\hline 80 ou mais & $0,83(0,67-1,02)$ & & $0,75(0,61-0,93)$ & \\
\hline Escolaridade em anos de estudo $(n=1.456)$ & & $<0,001$ & & $<0,001$ \\
\hline$\geq 12$ & 1,00 & & 1,00 & \\
\hline $9-11$ & $1,86(1,35-2,57)$ & & $1,48(1,10-1,98)$ & \\
\hline $5-8$ & $2,73(1,97-3,78)$ & & $1,81(1,33-2,46)$ & \\
\hline$\leq 4$ & $3,42(2,53-4,62)$ & & $1,83(1,38-2,42)$ & \\
\hline Renda familiar per capita $(n=1.466)$ & & $<0,001$ & & $<0,001$ \\
\hline Quartil 4 & 1,00 & & 1,00 & \\
\hline Quartil 3 & $2,58(1,97-3,38)$ & & $1,83(1,44-2,33)$ & \\
\hline Quartil 2 & $3,78(2,95-4,83)$ & & $2,14(1,72-2,66)$ & \\
\hline Quartil 1 & $3,75(2,93-4,80)$ & & $2,14(1,75-2,67)$ & \\
\hline Possui cuidador $(n=1.465)$ & & 0,001 & & NS \\
\hline Não & 1,00 & & & \\
\hline Sim & $1,31(1,12-1,52)$ & & & \\
\hline Capacidade funcional $(n=1.469)$ & & $<0,001$ & & NS \\
\hline Ausência de dependência & 1,00 & & & \\
\hline Dependência leve & $1,01(0,84-1,22)$ & & & \\
\hline Dependência moderada/grave & $1,45(1,24-1,69)$ & & & \\
\hline Doença crônica $(n=1.447)$ & & $<0,001$ & & $<0,001$ \\
\hline $0-1$ & 1,00 & & 1,00 & \\
\hline $2-4$ & $1,39(1,15-1,68)$ & & $1,23(1,05-1,45)$ & \\
\hline 5 ou mais & $1,76(1,44-2,15)$ & & $1,34(1,14-1,59)$ & \\
\hline Plano de saúde $(n=1.466)$ & & $<0,001$ & & $<0,001$ \\
\hline $\operatorname{Sim}$ & 1,00 & & 1,00 & \\
\hline Não & $2,33(2,01-2,69)$ & & $1,68(1,49-1,89)$ & \\
\hline Consulta médica nos últimos 3 meses $(n=1.466)$ & & $<0,001$ & & $<0,001$ \\
\hline Sim & 1,00 & & 1,00 & \\
\hline Não & $0,69(0,57-0,82)$ & & $0,75(0,64-0,88)$ & \\
\hline Número de medicamentos utilizados $(n=1.466)$ & & $<0,001$ & & $\mathrm{E}$ \\
\hline 1 & 1,00 & & & \\
\hline $2-3$ & $1,05(0,81-1,36)$ & & & \\
\hline $4-5$ & $1,37(1,05-1,77)$ & & & \\
\hline 6 ou mais & $1,71(1,31-2,23)$ & & & \\
\hline
\end{tabular}

E: excluído por ser uma variável colinear com a variável "doença crônica"; IC95\%: intervalo de 95\% de confiança; NS: valor de p não significativo ou > 0,05; RPa: razão de prevalência na análise ajustada; RPb: razão de prevalência na análise bruta. 
Prevalência e fatores associados ao acesso a medicamentos

O presente estudo demonstrou uma alta prevalência de acesso a medicamentos prescritos aos idosos que residem no município de Florianópolis, com resultados acima dos obtidos em outros estudos populacionais 8,9,11,12.

Estudos demonstram que fatores socioeconômicos estão claramente ligados ao acesso a medicamentos, ou seja, nível econômico mais alto 14 e maior escolaridade apresentaram um maior acesso a medicamentos 9,11.

Florianópolis possui uma característica distinta em relação a outros municípios do país: um número crescente de aposentados de classe média migra para a cidade em busca de melhor qualidade de vida ${ }^{17}$. A renda média mensal obtida neste estudo (R\$ 1.392,00) mostra que os idosos possuem renda mais alta em relação à outros locais do Brasil 18. Além disso, 23,1\% dos idosos entrevistados possuíam como grau de escolaridade o nível superior, resultado também mais alto que no restante do país 18. Essas características facilitam o acesso aos medicamentos, por aumento da possibilidade financeira de obtê-los e pelo esclarecimento da importância da realização do tratamento prescrito 19 .

No presente estudo, a renda e a escolaridade não estiveram associadas ao desfecho, levando a concluir que a população é homogênea em relação à capacidade de aquisição dos medicamentos prescritos. Além disso, a utilização de medicamentos prescritos por médico/dentista é relativamente maior em indivíduos com melhor nível econômico 20 , e nesse estudo somente foram analisados os casos de indivíduos que utilizaram pelo menos um dos medicamentos prescrito por médico/dentista, o que pode ter aumentado a população de maior renda na amostra.

Todavia, não se pode afirmar que esse acesso ocorra de forma regular, uma vez que uma parcela da população referiu acesso parcial ou nenhum acesso aos medicamentos de que necessitava no mês anterior à entrevista. Os maiores motivos para não obtenção do medicamento foram a falta de dinheiro para comprá-lo e a falta do medicamento na farmácia do SUS. O mesmo resultado foi obtido em estudo que avaliou as condições de saúde dos idosos do Município de Florianópolis 17.

Vários estudos já demonstraram que, quanto maior o número de doenças crônicas e de utilização dos serviços de saúde, maior é a necessidade de utilização de medicamentos 11,19,20,21,22,23. Porém neste estudo, as associações mostraram que quanto maiores as necessidades em saúde do idoso (maior número de doenças crônicas, maior grau de dependência e maior necessidade de consulta ao médico), menor é a proporção de acesso aos medicamentos que lhe são prescritos. O que pode explicar esse resultado é que, com o aumento da necessidade de utilização de um maior número de medicamentos, a probabilidade de não obter todos os medicamentos prescritos é maior.

\section{Prevalência e fatores associados ao acesso a medicamentos pelo SUS}

Os resultados da análise da proporção de idosos que utilizam o SUS para obter medicamentos e seus fatores associados demonstram que o perfil socioeconômico do usuário do SUS é, hegemonicamente, composto por estratos populacionais de baixa renda e escolaridade, onde está a maior concentração de pessoas com cor de pele considerada parda e preta. Porém, a cor da pele continuou associada a um maior acesso a medicamentos pelo SUS mesmo após o ajuste para as variáveis socioeconômicas, resultado este que pode indicar que existem diferenças de inserção social nesses indivíduos não explicadas pelas variáveis socioeconômicas, o que causa uma desigualdade na forma de obtenção de medicamentos.

O SUS tem como diretriz ser universal e igualitário, disponível para toda a população, independentemente de condição socioeconômica. Porém, a existência de grandes desigualdades sociais e a presença de um sistema privado de saúde faz com que os serviços do SUS sejam utilizados preferencialmente por parcelas da população não cobertas pelo sistema privado, que apresentam condições socioeconômicas desfavorecidas 24 .

A universalização da saúde, apesar de ser um avanço histórico nas políticas sociais no Brasil, imprime uma sobrecarga de demanda de grande impacto sobre a organização dos serviços. O que se observa é uma quantidade crescente de necessidade não acompanhada do aporte financeiro equivalente, o que gera consequências no tempo de oferta de alguns serviços.

Políticas efetivamente universais precisam atender às expectativas de qualidade de toda a sociedade, porém os estratos superiores da estrutura social ainda encontram seus interesses mais bem supridos no sistema privado. Os prováveis motivos seriam o desconhecimento sobre a disponibilidade de medicamentos pelo SUS, ou por diferenças de prescrição entre médicos privados e do SUS (medicamentos prescritos que são da mesma classe terapêutica, mas não estão disponíveis na Relação Municipal de Medicamentos). 
A maioria dos indivíduos que obtém medicamentos pelo SUS não possui plano de saúde e a proporção aumenta quanto maior o número de doenças crônicas. Portanto, percebe-se que quem possui maiores necessidades em saúde também utiliza mais o SUS para obter medicamentos, fato já observado em outro estudo que avaliou a utilização de serviços de saúde 24 .

\section{Conclusão}

Os resultados dessas duas análises - a alta prevalência de acesso a medicamentos, juntamente com o perfil social dos idosos que utilizam o SUS para obter seus medicamentos - levam a crer que o SUS vem cumprindo seu papel na tentativa de universalização do acesso à saúde, e nesse caso, aos medicamentos, pois quem não possui condições de comprar seus medicamentos está suportado pelo SUS para conseguir obtê-los. A universalização do acesso à saúde proposta pelo SUS vem incorporando estratos sociais antes excluídos, que não conseguiriam obter seus medicamentos através da compra desses 25 .

\section{Resumo}

O presente estudo tem por objetivo descrever a prevalência, fatores associados e a forma de acesso aos medicamentos prescritos aos idosos residentes na área urbana do Município de Florianópolis, Santa Catarina, Brasil. Foi realizado estudo transversal populacional de base domiciliar em amostra de 1.469 indivíduos (taxa não-resposta 10,8\%). Os dados foram analisados, por meio de regressão de Poisson, sob duas perspectivas: do acesso a medicamentos em geral e do acesso a medicamentos pelo SUS. Os resultados foram, respectivamente, 95,8\% (IC95\%: 94,7-96,8) e 50,3\% (IC95\%: 47,7-52,8). Observou-se que ocorre menor acesso a medicamentos quanto maior a dependência funcional, maior o número de doenças crônicas e quando há realização de consulta médica. Acessam mais medicamentos pelo SUS indivíduos de cor da pele parda ou negra, menor idade, pior escolaridade, pior renda, maior número de doenças crônicas, ausência de plano de saúde privado e consulta médica nos últimos três meses. Estes resultados mostram que o SUS vem cumprindo seu papel na universalização do acesso aos medicamentos em Florianópolis.

Idoso; Acesso aos Serviços de Saúde; Uso de Medicamentos; Sistema Único de Saúde; Estudos Transversais
Por outro lado, considerando que se trata de uma política universal, onde todos têm direito ao acesso, estes dados evidenciam a dificuldade de se atingir padrões de efetiva universalidade em contextos de marcada desigualdade de renda. Isso ainda ocorre, pois a parcela da população com melhor poder aquisitivo tem a opção de escolher onde ter acesso à saúde, e encontram respostas razoáveis às suas demandas de saúde na área privada.

O principio da equidade reconhece que os indivíduos são diferentes, e que tratamentos iguais nem sempre são equitativos. Portanto, estratos sociais mais desfavorecidos necessitam de maior atenção das políticas públicas para a garantia de acesso aos medicamentos.

A universalização de acesso a serviços de saúde, em particular o acesso a medicamentos, está em fase de transição e deve ser alvo de constante aprimoramento. Enquanto o sistema público não tiver condições de suprir a crescente demanda das necessidades em saúde de toda a população, com a qualidade necessária, esta segmentação entre as formas de obtenção de acesso à saúde entre as classes sociais continuará evidente.

\section{Colaboradores}

M. M. Aziz contribuiu para a concepção, planejamento, análise, interpretação dos dados, elaboração do rascunho, revisão crítica do conteúdo, e aprovação da versão final do manuscrito. M. C. Calvo contribuiu para a concepção, análise e interpretação dos dados, elaboração do rascunho e aprovação final do manuscrito. I. J. C. Schneider e A. J. Xavier contribuíram para o planejamento, análise dos dados; revisão e aprovação do manuscrito. E. d'Orsi contribuiu para a concepção, planejamento, análise e interpretação dos dados, elaboração do rascunho e aprovação final do manuscrito.

\section{Agradecimentos}

Os autores agradecem aos técnicos do Instituto Brasileiro de Geografia e Estatística (IBGE) e da Secretaria Municipal de Saúde de Florianópolis pelo auxílio na operacionalização deste estudo. À Dra. Nilza Nunes da Silva (Faculdade de Saúde Pública/USP), pelas contribuições com os procedimentos de amostragem e aos dicentes dos Programas de Pós-graduação em Saúde Coletiva, Educação Física e Nutrição (UFSC), que realizaram a função de supervisores do estudo. 


\section{Referências}

1. Instituto Brasileiro de Geografia e Estatística. Indicadores sociodemográficos e de saúde no Brasil - 2009. Rio de Janeiro: Instituto Brasileiro de Geografia e Estatística; 2009. (Estudos e Pesquisas - Informação Demográfica e Socioeconômica, 25).

2. Rozenfeld S. Prevalência, fatores associados e mau uso de medicamentos entre os idosos: uma revisão. Cad Saúde Pública 2003; 19:717-24.

3. Ministério da Saúde. Política Nacional de Medicamentos. Brasília: Ministério da Saúde; 1998.

4. Coordenação-Geral de Apoio à Gestão Descentralizada, Departamento de Apoio à Descentralização, Secretaria Executiva, Ministério da Saúde. Diretrizes operacionais dos Pactos pela Vida, em Defesa do SUS e de Gestão. Brasília: Ministério da Saúde; 2006.

5. Ministério da Saúde/Organização Pan-Americana da Saúde/Organização Mundial da Saúde. Avaliação da assistência farmacêutica no Brasil: estrutura, processo e resultados. Brasília: Ministério da Saúde; 2005.

6. Oliveira MA, Bermudez JAZ, Osorio-de-Castro CGS. Assistência farmacêutica e acesso a medicamentos. Rio de Janeiro: Editora Fiocruz; 2007.

7. Lima-Costa MF, Barreto SM, Giatti L. Condições de saúde, capacidade funcional, uso de serviços de saúde e gastos com medicamentos da população idosa brasileira: um estudo descritivo baseado na Pesquisa Nacional por Amostra de Domicílios. Cad Saúde Pública 2003; 19:735-43.

8. Oliveira BB. Investigações de acesso a medicamentos em nível domiciliar: um estudo comparativo de iniciativas realizadas no Brasil entre $2002 \mathrm{e}$ 2004 [Tese de Doutorado]. Rio de Janeiro: Escola Nacional de Saúde Pública Sergio Arouca, Fundação Oswaldo Cruz; 2007.

9. Paniz VMV, Fassa AG, Facchini LA, Bertoldi AD, Piccini RX, Tomasi E, et al. Acesso a medicamentos de uso contínuo em adultos e idosos nas regiões Sul e Nordeste do Brasil. Cad Saúde Pública 2008; 24:267-80.

10. Luz TCB, Loyola Filho AI, Lima-Costa MF. Estudo de base populacional da subutilização de medicamentos por motivos financeiros entre idosos na Região Metropolitana de Belo Horizonte, Minas Gerais, Brasil. Cad Saúde Pública 2009; 25:1578-86.

11. Carvalho MF, Pascom ARP, Souza-Júnior PRB, Damacena GN, Szwarcwald CL. Utilization of medicines by the Brazilian population, 2003. Cad Saúde Pública 2005; 21 Suppl:S100-8.

12. Paniz VMV, Fassa AG, Facchini LA, Piccini RX, Tomasi E, Thumé E, et al. Free access to hypertension and diabetes medicines among the elderly: a reality yet to be constructed. Cad Saúde Pública 2010; 26:1163-74.

13. Instituto Brasileiro de Geografia e Estatística. Estimativas populacionais para o TCU. Estimativas da população para 1o de julho de 2009. http://www. ibge.gov.br/home/estatistica/populacao/estima tiva2009/POP2009_DOU.pdf (acessado em 03/ Dez/2009).
14. Programa das Nações Unidas para o Desenvolvimento. Atlas do desenvolvimento humano no Brasil, 2003. http://www.pnud.org.br/atlas/ (acessado em 03/Dez/2009).

15. Instituto Brasileiro de Geografia e Estatística. Censo 2000. http://www.ibge.gov.br/home/estatisti ca/populacao/censo2000/default.shtm (acessado em 01/Jul/2008).

16. Rosa TEC, Benício MHD, Latorre MRDO, Ramos LR. Fatores determinantes da capacidade funcional entre idosos. Rev Saúde Pública 2003; 37:40-8.

17. Benedetti TB, Petroski EL, Gonçalves LT. Condições de saúde nos idosos de Florianópolis. ACM Arq Catarin Med 2006; 35:44-51.

18. Instituto Brasileiro de Geografia e Estatística. Perfil dos idosos responsáveis pelos domicílios no Brasil - 2000. Rio de Janeiro: Instituto Brasileiro de Geografia e Estatística; 2002. (Estudos e Pesquisas - Informação Demográfica e Socioeconômica, 9).

19. Loyola Filho AI, Uchôa E, Firmo JOA, Lima-Costa MF. Estudo de base populacional sobre o consumo de medicamentos entre idosos: Projeto Bambuí. Cad Saúde Pública 2005; 21:545-53.

20. Coelho Filho JM, Marcopito LF, Castelo A. Perfil de utilização de medicamentos por idosos em área urbana do Nordeste do Brasil. Rev Saúde Pública 2004; 38:557-64.

21. Loyola Filho AI, Uchoa E, Lima-Costa MF. Estudo epidemiológico de base populacional sobre uso de medicamentos entre idosos na Região Metropolitana de Belo Horizonte, Minas Gerais, Brasil. Cad Saúde Pública 2006; 22:2657-67.

22. Loyola Filho AI, Uchoa E, Firmo JOA, Lima-Costa MF. Influência da renda na associação entre disfunção cognitiva e polifarmácia: Projeto Bambuí. Rev Saúde Pública 2008; 42:89-99.

23. Ribeiro AQ, Rozenfeld S, Klein CH, César CC, Acurcio FA. Inquérito sobre uso de medicamentos por idosos aposentados, Belo Horizonte, MG. Rev Saúde Pública 2008; 42:724-32.

24. Ribeiro MCSA, Barata RB, Almeida MF, Silva ZP. Perfil sociodemográfico e padrão de utilização de serviços de saúde para usuários e não-usuários do SUS - PNAD 2003. Ciênc Saúde Coletiva 2006; 11:1011-22.

25. Fahel M. Desigualdades em saúde na população brasileira: uma análise comparada a partir das Pesquisas Nacionais por Amostra de Domicílios 1998 e 2003, com foco em Minas Gerais. In: XIII Congreso Internacional del CLAD sobre la Reforma del Estado y de la Administración Pública. Buenos Aires: Documento Libre; 2008. p. 1-28.

Recebido em 03/Mar/2011

Versão final reapresentada em 07/Jun/2011

Aprovado em 27/Jun/2011 